\title{
Dynamic Modeling of Micro-Satellite Spartnik's Attitude
}

\author{
Brian M. Menges and Carlos A. Guadiamos \\ San José State University \\ Dr. Henry J. Pernicka \\ Department of Mechanical and Aerospace Engineering \\ One Washington Square, San José CA 95192-0087
}

Spartnik is a micro-satellite under construction at San Jose State University. In order to control the satellite and ensure payloads and antenna are oriented properly a passive attitude control system has been developed. Like some other micro-satellites, Spartmik will combine spin stabilization with magnetic stabilization. Thus, Spartmik will "spin" due to solar radiation pressure and perform a controlled "tumble" due to the permanent magnets aligning with the magnetic field of the Earth. Concerns have been raised about the gyroscopic effect due to the spin of the satellite. A program has been developed that numerically integrates Euler's form of the equations of motion in order to explore the gyroscopic effect and finalize the hardware design of Spartmik. Simulations have been performed that reveal the dynamic response of Spartikik under a mumber of conditions. This paper includes a detailed discussion of these results.

\section{Introduction}

Spartnik is a micro-satellite being designed and built by senior design students at San José State University. The goal of the project is to educate the students on how to design, manufacture, assemble, test, and operate a micro-satellite. Spartnik will be launched into a low Earth orbit (LEO) and will carry three experimental payloads: a communications package (which will transmit on amateur radio frequencies), a color digital camera, and a micro-meteorite impact detector (MMID).

A series of requirements placed on the design team are as follows: to (1) meet the secondary payload constraints for as many launch vehicles as possible, (2) be designed for multiple orbit configurations, ranging from 300 to $1000 \mathrm{~km}$ altitude and asi inclination of greater than $30^{\circ}$, (3) have an operational life of at least two years, (4) use as many acceptable non-space rated parts as possible, (5) use passive and autonomous systems where possible, and (5) use communications systems that use HAM frequencies for uplink and downlink. The ground station, used to communicate with Spartnik, will be located at San José State University. This places the requirement that the orbital inclination be greater than $30^{\circ}$.

When in orbit satellites must be oriented about their center of mass correctly to achieve their mission objectives. Satellite components such as antenna and payloads must be pointed to within a tolerable range at locations on the Earth's surface. Spartnik is no exception. Therefore, a passive control system that will achieve this requirement is needed.

\section{Basic Attitude Control Design}

The passive attitude control system selected for Spartnik is a combination of spin and magnetic field stabilization. This design is closely based upon the designs used by WeberSat, AMSAT, and other microsatellites. Permanent magnets mounted in the outer shell of Spartnik will align the satellite with the Earth's magnetic field as it moves on its orbit. This will have the effect of causing the satellite to "tumble" twice per orbit. The magnets will be oriented such that Spartnik's top panel, where the camera lens is mounted, will face Earthward when over the northern hemisphere and out to space when over the southern hemisphere. This tumble is depicted for one fourth of an orbit below:

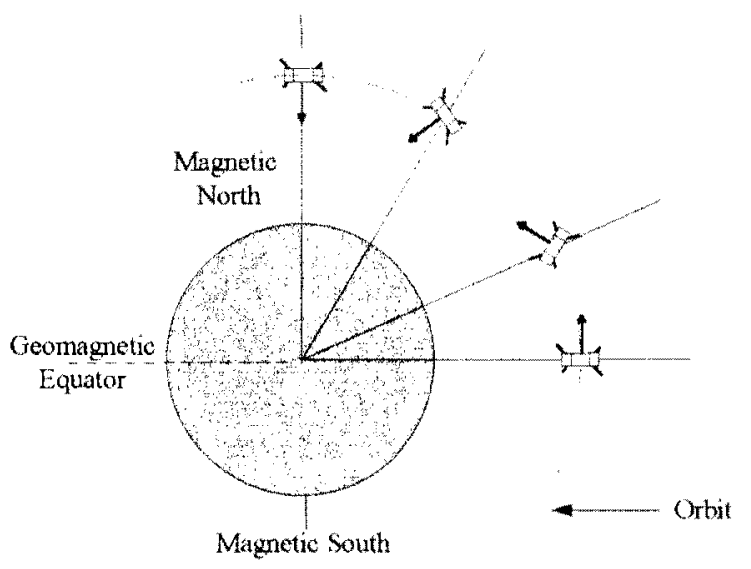

Figure 1: Spartnik Attitude for One-Fourth of an Orbit

Spin stabilization is used to prevent any disturbance torques from creating excessive nutation as well as provide uniform thermal distribution and "wear" on the solar panels. Spin is achieved through the use of solar 
pressure paddles (SPP), which also serve the dual purpose as uplink and downlink antennas. Solar pressure on these paddles produces a torque causing the satellite to spin. However, without any dissipative forces, the solar pressure would continue to accelerate the spin of Spartnik. To maintain a target (constant) spin rate hysteresis rods have been added to the design. Four hysteresis rods are attached to a plate which lies in a plane normal to the spin vector. The rotation of these rods perpendicular to the magnetic field will dissipate spin energy and maintain the target spin rate. The location of the hardware described above is depicted in Figure 2 .

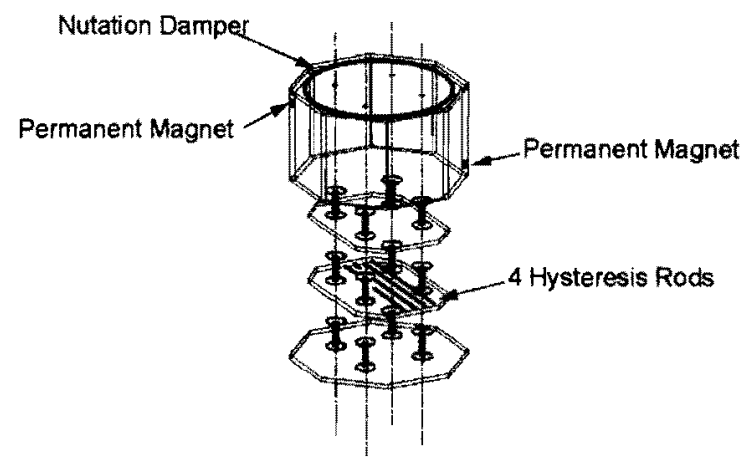

Figure 2: Location of Attitude Control Hardware

Concerns have been raised about the dynamic interaction of the spin and the induced "tumble," known as the gyroscopic effect. The gyroscopic effect is the tendency of a spinning rigid body subjected to an applied torque to rotate about the axis perpendicular to the plane formed by the spin vector and the axis about which the torque is applied. If the spin is too great it may induce enough gyroscopic effect so as to cause undesirable nutation, or at worst, to prevent the satellite from tumbling correctly. The strength of the magnets is also important. If the magnets are too weak the satellite will not lock onto the Earth's magnetic field lines and will not experience the desired tumble or point toward Earth when needed ${ }^{2}$. Therefore, the spin rate and magnets must be chosen carefully. Furthermore, because many micro-satellite projects, including Spartnik, are financially challenged they must accept a donated launch as a secondary payload. The final orbit, therefore, is not known and the attitude control design must accommodate a wide range of candidate orbits.

In order to address these concerns and make recommendations about the magnets strength simulation software was needed to model the attitude of Spartnik while in orbit. The simulation method chosen was the use of numerical integration to solve the equations of motion (EOMs). A simulation would allow the investigation of different orbits (altitude and inclination) before launch thereby permitting changes in design to best suite a wide range of possible orbits. The overall purpose of the simulation, however, is to determine the size and strength of the permanent magnets to be used on the satellite and, since the exact spin rate has yet to be chosen, investigate the gyroscopic effect.

\section{Dynamic Model}

The initial step in the design of any simulation is to determine what the purpose of the simulation will be, what needs to be modeled and what information is desired. In the case of Spartnik, the goal of the simulation is threefold. The primary goal is to give some insight into whether the "spin" and "tumble" that Spartnik will experience in orbit will interact with each other, either by disrupting or canceling each other out. The fear being that, when spinning, Spartnik will experience the gyroscopic effect and the induced tumble will interact with this spin and cause Spartnik to nutate in an undesirable manner. The simulation will verify if this effect will indeed happen and if so what can be done to minimize it. A second goal is to determine when one of the payloads, the color digital camera, is pointed toward the Earth for the purpose of meaningful picture taking. The third and final goal of the simulation is to generate an attitude profile of Spartnik over the course of one orbit. Such a profile could then be compared to the real attitude data once the satellite is in orbit.

\section{Physical and Geometric Properties of Spartnik}

Before the simulation model is described an understanding of the physical properties of Spartnik is important. Spartnik's configuration is a regular octagon measuring $17 \mathrm{~cm}$ on a side and $25 \mathrm{~cm}$ in height. The final flight vehicle mass is estimated at $40 \mathrm{~kg}$. Solar panels are mounted on each face of Spartnik's outer shell and will provide six Watts of power. Spartnik's general shape is depicted in Figure 3.

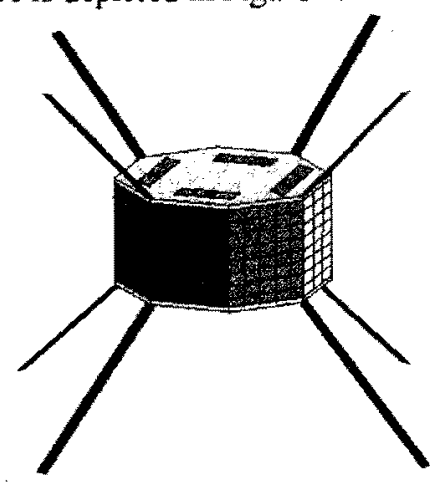

Figure 3: Micro-satellite Spartnik 


\section{Definition of Frames/ Rotations}

The first step to developing the simulation is to define a set of coordinates and coordinate frames in which the dynamic model will be developed. Three coordinate frames were chosen to model the dynamics of Spartnik: an inertial frame, a rotating frame, and a body fixed (also rotating) frame.

\section{The Inertial (I) Frame $X-Y-Z$}

The first frame defined is an inertial frame, labeled $\mathbf{X}-\mathbf{Y}-\mathbf{Z}$ and hereafter called simply the inertial frame. The origin is located at the center of the Earth with the $\mathbf{X}$ axis defined to point in the direction of the Vernal Equinox, $\mathbf{Z}$ pointing north and $\mathbf{Y}$ completing the right handed frame. This frame is used primarily to calculate the latitude and longitude of Spartnik's center of mass as it moves along its orbit. The process by which the spacecraft latitude and longitude are calculated is described later

\section{The Local Rotating (R) Frame $x-y-z$}

Next a rotating frame, labeled $\mathbf{x}-\mathbf{y}-\mathbf{z}$ and hereafter referred to as simply the rotating frame, is defined with its origin centered on Spartnik's center of mass. The $\mathbf{x}$ axis points in the direction of motion in the plane of the orbit, $\mathbf{z}$ is nadir pointing (i.e. toward the center of the Earth), and $\mathbf{y}$ completes the right handed frame. Although this frame is defined with its origin at the center of mass and rotates around the orbital plane with Spartnik, it is not fixed in the body of the satellite. Therefore, the $\mathrm{z}$ axis will always be nadir pointing. The inertial and rotating frames are graphically depicted in Figure 4

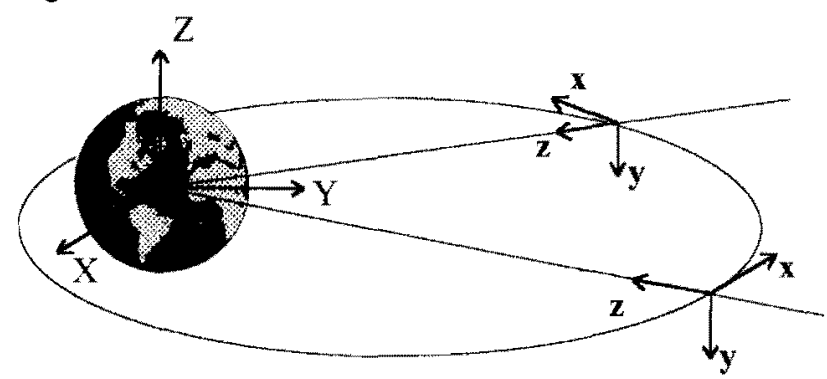

Figure 4: The Inertial and Rotating Frames

\section{The Body (B) Frame $b_{1}-b_{2}-b_{3}$}

A third frame, labeled $\mathbf{b}_{1}-\mathbf{b}_{2}-\mathbf{b}_{3}$ and hereafter called the body frame, is needed. The origin of this frame is centered on Spartnik's center of mass and is defined such that $\mathbf{b}_{1}$ points outward normal to Panel 3 , $\mathbf{b}_{2}$ points outward normal to Panel 1 and $\mathbf{b}_{3}$ points outward normal to the top Panel. This frame is graphically represented below in

Figure 5 .

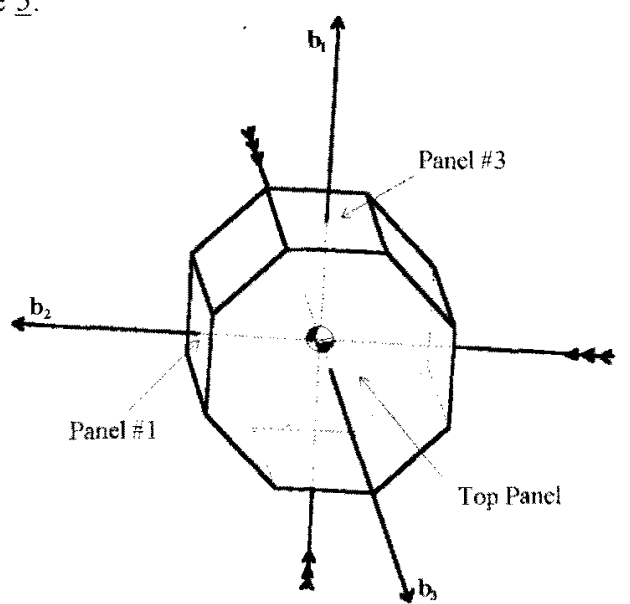

Figure 5: The Body Frame

The body frame is defined such that it is fixed in the body of Spartnik and thus will be used to determine the orientation of Spartnik with respect to the rotating frame. Since the $\mathbf{z}$ axis is always nadir pointing the offset of the $\mathbf{b}_{3}$ axis from nadir can easily be used to measure the performance of the attitude control system. If Spartnik's attitude control system is working as designed this offset angle will be small when over the northern hemisphere. Therefore, one of the primary goals of the simulation, namely whether the camera is pointed Earthward when over the northern hemisphere, can be determined quickly. The end goal of the simulation is to generate a time history of the orientation of the body frame with respect to the rotating frame.

In order to relate the rotating frame to the body frame a $1-2-3$ body Euler rotation is performed. Initially, the body frame can be assumed to be aligned with the rotating frame, that is $\mathbf{b}_{\mathbf{1}}$ aligned along the $\mathbf{x}$ axis, $\mathbf{b}_{\mathbf{2}}$ along the $\mathbf{y}$-axis, and $\mathbf{b}_{\mathbf{3}}$ along the $\mathbf{z}$-axis. First, the body frame is pitched $\phi$ degrees about the $\mathbf{x}$ axis. Next, the resulting intermediate frame is pitched $\theta$ degrees about the $\mathbf{y}^{\prime}$ axis and finally yawed $\psi$ degrees about the $\mathbf{z}$ " axis. These three rotations, $\phi, \theta$ and $\psi$, are shown in Figure 6.

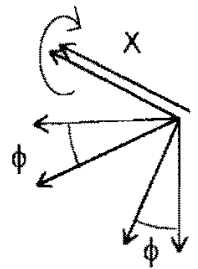

Roll $\phi$ along $X$ axis

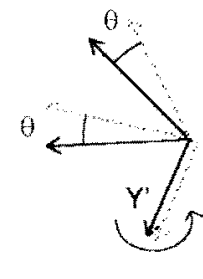

Pitch $\theta$ along $Y^{\prime}$ axis

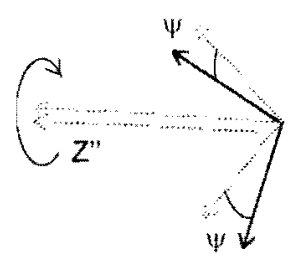

Yaw $\psi$ along $Z^{\prime \prime}$ axis
Figure 6: Body 1-2-3 Rotation 
Combining the results of these three rotations leads to a direction cosine matrix allowing the transformation of any vector from the rotating frame to the body frame. The matrix becomes:

$$
\left\{\begin{array}{l}
\mathbf{b}_{\mathbf{1}} \\
\mathbf{b}_{\mathbf{2}} \\
\mathbf{b}_{\mathbf{3}}
\end{array}\right\}=\left[\begin{array}{ccc}
C_{\theta} C_{\psi} & S_{\phi} S_{\theta} C_{\psi}+C_{\phi} S_{\psi /} & -C_{\phi} S_{\theta} C_{\psi}+S_{\phi} S_{\psi} \\
-C_{\theta} S_{\psi} & -S_{\phi} S_{\theta} S_{\phi}+C_{\phi} C_{\psi} & C_{\phi} S_{\theta} S_{\psi}+S_{\phi} C_{\psi} \\
S_{\theta} & -S_{\phi} C_{\theta} & C_{\phi} C_{\theta}
\end{array}\right]\left\{\begin{array}{l}
\mathbf{x} \\
\mathbf{y} \\
\mathbf{z}
\end{array}\right\}
$$

\section{Derivations of the Equations of Motion}

With a set of frames defined, a means to rotate between them, and the objectives of the simulation in mind the EOMs can now be derived. Numerical integration of these EOMs will give a time history of the orientation of Spartnik. A few assumptions are built into the simulation. First, an early estimate of the mass distribution of Spartnik generated the following moments of inertia:

Table 1: Estimated Moments of Inertia

\begin{tabular}{|l|l|}
\hline$I_{x x}=0.30393 \mathrm{~kg} \cdot \mathrm{m}^{2}$ & $I_{\mathrm{xy}}=0.00009 \mathrm{~kg} \cdot \mathrm{m}^{2}$ \\
\hline $\mathrm{I}_{\mathrm{yy}}=0.32415 \mathrm{~kg} \cdot \mathrm{m}^{2}$ & $\mathrm{I}_{\mathrm{xz}}=-0.00044 \mathrm{~kg} \cdot \mathrm{m}^{2}$ \\
\hline $\mathrm{I}_{\mathrm{zz}}=0.48614 \mathrm{~kg} \cdot \mathrm{m}^{2}$ & $\mathrm{I}_{\mathrm{yz}}=-0.00315 \mathrm{~kg} \cdot \mathrm{m}^{2}$ \\
\hline
\end{tabular}

Although these values are not finalized they show that the satellite's body axes, as defined previously, can be assumed to be along principle axes. This assumption allows the derivation of the EOMs to be simplified, using the fundamental equation from Newtonian mechanics

$$
\mathbf{M}=\frac{{ }^{l} d \mathbf{H}}{d t}=\frac{d}{d t}\left\{[I]^{\mathrm{I}} \boldsymbol{\omega}^{\mathrm{B}}\right\}
$$

where

$\mathbf{M}=$ sum of the external moments about the center of mass $(\mathrm{N} \cdot \mathrm{m})$

${ }^{\mathrm{I}} \mathrm{d} \mathbf{H} / \mathrm{dt}=$ time derivative of the angular momentum about the center of mass relative to an inertial frame $\left(\mathrm{kg} \cdot \mathrm{m}^{2} / \mathrm{s}\right)$

[I] = inertia matrix about the center of mass, assumed constant for Spartnik $\left(\mathrm{kg} \cdot \mathrm{m}^{2}\right)$
${ }^{\mathrm{I}} \omega^{\mathrm{B}}=$ angular velocity vector of the body frame relative to an inertial frame (radians/s)

Breaking down the overall equation into its different components using Euler's form of equation (2) gives

$$
\begin{aligned}
& M_{1}=I_{x x} \dot{\omega}_{x}+\left(I_{z z}-I_{y y}\right) \omega_{y} \omega_{z} \\
& M_{2}=I_{y} \dot{\omega}_{y}+\left(I_{x x}-I_{z z}\right) \omega_{x} \omega_{z} \\
& M_{3}=I_{z z} \dot{\omega}_{z}+\left(I_{y y}-I_{z z}\right) \omega_{x} \omega_{y}
\end{aligned}
$$

where

$M_{1}=$ sum of the externally applied moments about the $\mathbf{x}$ axis $(\mathrm{N} \cdot \mathrm{m})$

$\mathrm{M}_{2}=$ sum of the externally applied moments about the $\mathrm{y}-$ axis $(\mathrm{N} \cdot \mathrm{m})$

$M_{3}=$ sum of the externally applied moments about the $z$ axis $(\mathrm{N} \cdot \mathrm{m})$

$I_{x x}=$ moment of inertia about the $x$-axis $\left(\mathrm{kg} \cdot \mathrm{m}^{2}\right)$

$I_{y y}=$ moment of inertia about the $y$-axis $\left(\mathrm{kg} \cdot \mathrm{m}^{2}\right)$

$\mathrm{I}_{z z}=$ moment of inertia about the $\mathrm{z}$-axis $\left(\mathrm{kg} \cdot \mathrm{m}^{2}\right)$

Note $I_{x y}, I_{x z}$, and $I_{y z}$ are approximated as zero.

Solving equations $(3 \mathrm{a}),(3 \mathrm{~b})$, and (3c) for $\dot{\omega}_{x}, \dot{\omega}_{y}, \dot{\omega}_{z}$ leads to

$$
\begin{aligned}
& \dot{\omega}_{x}=\left(\frac{1}{I_{x x}}\right)\left[M_{x}-\left(I_{z z}-I_{y y}\right) \omega_{y} \omega_{z}\right] \\
& \dot{\omega}_{y}=\left(\frac{1}{I_{y y}}\right)\left[M_{y}-\left(I_{x x}-I_{z z}\right) \omega_{x} \omega_{z}\right] \\
& \dot{\omega}_{z}=\left(\frac{1}{I_{z z}}\right)\left[M_{z}-\left(I_{y y}-I_{x x}\right) \omega_{x} \omega_{y}\right]
\end{aligned}
$$

These equations are only valid if the angular velocity is described with respect to an inertial frame. The angular velocity of the body frame with respect to the inertial frame can be expressed as

$$
{ }^{I} \boldsymbol{\omega}^{B}={ }^{I} \boldsymbol{\omega}^{R}+{ }^{R} \boldsymbol{\omega}^{B}
$$

Expressed in body frame coordinates this becomes

$$
{ }^{1} \omega^{B}=\omega_{x} \mathbf{b}_{1}+\omega_{y} \mathbf{b}_{2}+\omega_{z} \mathbf{b}_{3}
$$


Assuming a circular polar orbit with constant orbital speed, the angular velocity of the rotating frame with respect to the inertial frame is simply

$$
{ }^{t} \boldsymbol{\omega}^{R}=-n \mathbf{y}
$$

where " $n$ " is the mean orbital motion equal to the angular rate of Spartnik moving on its orbit or

$$
n=\sqrt{\frac{\mu}{a^{3}}}
$$

where

$\mu=$ geocentric gravitational constant $\left(3.986 \times 10^{5}\right.$

$\mathrm{km}^{3} / \mathrm{sec}^{2}$ )

$a=$ semi-major axis of orbit $(\mathrm{km})$

Converting equation (7) into body frame coordinates using the direction cosine matrix from equation (1) leads to

$$
\begin{aligned}
{ }^{1} \boldsymbol{\omega}^{R}= & \left(-n S_{\phi} S_{\theta} C_{\psi}-n C_{\phi} S_{\psi}\right) \mathbf{b}_{1}+ \\
& \left(n S_{\phi} S_{\phi} S_{\psi}-n C_{\phi} C_{\psi}\right) \mathbf{b}_{2}+\left(n S_{\phi} C_{\psi}\right) \mathbf{b}_{3}
\end{aligned}
$$

The angular velocity of the body frame with respect to the rotating frame, ${ }^{\mathrm{R}} \boldsymbol{\omega}^{\mathrm{B}}$, can be expressed as follows

$$
{ }^{R} \omega^{B}=\dot{\phi} \mathbf{x}+\dot{\theta} \mathbf{y}^{\prime \prime}+\dot{\psi b} \mathbf{b}_{3}
$$

where $\dot{\phi}, \dot{\theta}$, and $\dot{\psi}$ are as described in Figure 6 . This equation, expressed in the body frame, becomes

$$
{ }^{R} \omega^{B}=\omega_{1} \mathbf{b}_{1}+\omega_{2} \mathbf{b}_{2}+\omega_{3} \mathbf{b}_{3}
$$

where

$$
\begin{aligned}
& \omega_{1}=\dot{\theta} \sin \psi+\dot{\phi} \cos \theta \cos \psi \\
& \omega_{2}=\dot{\theta} \cos \psi-\dot{\phi} \cos \theta \sin \psi \\
& \omega_{3}=\dot{\phi} \sin \theta+\dot{\psi}
\end{aligned}
$$

Equation (10) gives an expression of the angular velocity of the body frame with respect to the rotating frame that can be (numerically) integrated with equation $(4 a-c)$ once the external moment components $\mathrm{M}_{\mathrm{x}}, \mathrm{M}_{\mathrm{y}}, \mathrm{M}_{\mathrm{z}}$ are specified. Substituting equations (6) and (9) into (5) and equating like-terms leads to the following three equations

$$
\begin{aligned}
& \omega_{1}=\omega_{x}+n S_{\phi} S_{\theta} C_{\psi}+n C_{\phi} S_{\psi} \\
& \omega_{2}=\omega_{y}-n S_{\phi} S_{\theta} S_{\psi}+n C_{\phi} C_{\psi} \\
& \omega_{3}=\omega_{z}-n S_{\phi} C_{\theta}
\end{aligned}
$$

Finally, solving equation (12) for $\dot{\phi}, \dot{\theta}$, and $\dot{\psi}$ leads to the following expressions for the angular velocities of Spartnik

$$
\begin{aligned}
& \dot{\phi}=\left(\frac{C_{\psi}}{C_{\theta}}\right) \omega_{1}-\left(\frac{S_{\psi}}{C_{\theta}}\right) \omega_{2} \\
& \dot{\theta}=S_{\psi} \omega_{1}+C_{\psi} \omega_{2} \\
& \dot{\psi}=\left(\frac{-S_{\theta} C_{\psi}}{C_{\theta}}\right) \omega_{1}+\left(\frac{S_{\theta} S_{\psi}}{C_{\theta}}\right) \omega_{2}+\omega_{3}
\end{aligned}
$$

Note that these expressions experience a singularity when $\theta$ equals 90 degrees. Additionally, $\theta$ is limited to a range of $-90^{\circ}$ to $+90^{\circ}$. These final three equations, along with the three equations (4a-c), can be numerically integrated to generate a time history of the orientation of Spartnik

\section{Modeling of External Moments}

Once the equations of motion and coordinate frames are identified and defined the external moments that will be acting on Spartnik need to be identified and modeled. Spartnik will experience at least four different torques while in orbit. These torques result from aerodynamic drag, solar radiation pressure, gravity gradient, and magnetic field effects ${ }^{3}$. Calculations show that the magnetic field interaction is three orders of magnitude greater than the other three torques ${ }^{4}$. Therefore, it is the first external moment to be modeled.

\section{Earth's Magnetic Field}

In order to model the interaction of the permanent magnets with the Earth's magnetic field a reliable model for the Earth's magnetic field is needed. The field model used is the International Geomagnetic Reference Field (IGRF) model. This model includes the main or core field without external sources, such as the interaction of the field with the solar wind. The field model is valid for altitudes up to 30,000 kilometers and for the years 1945 to $2000^{5}$. Two sets of magnetic field data with different resolutions have been obtained. One is a $5^{\circ}$ latitude by $5^{\circ}$ longitude grid and the other is a $10^{\circ}$ by $10^{\circ}$ grid, both compiled for a $1000 \mathrm{~km}$ altitude. Simulations can be run using the $10^{\circ}$ by $10^{\circ}$ grid for the 
purpose of debugging the code. Once the bugs are fixed, and one obtains trustworthy results, the grid definition can be increased to $5^{\circ}$ by $5^{\circ}$ for more accurate results.

In order to obtain the field vector for longitude and latitude values that lie within the grid points bilinear interpolation is used. Reference 7 describes the interpolation method used in this simulation. A description of the core field and a single block of the downloaded grid is pictured in Figure 7.

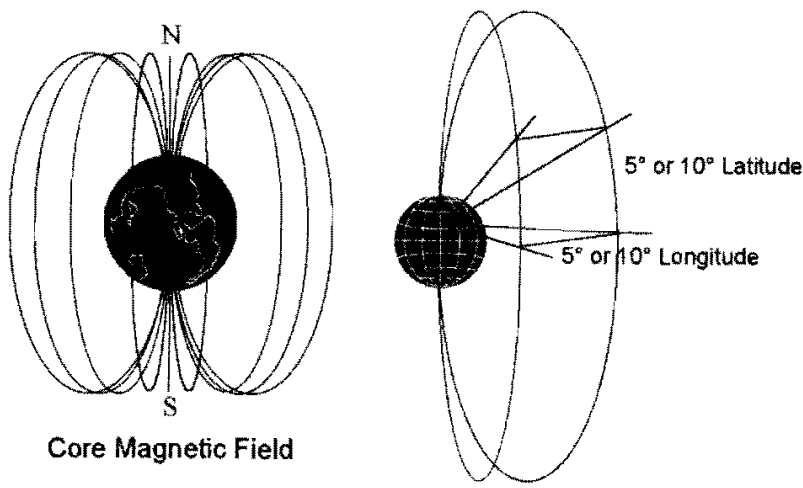

Figure 7: Core Magnetic Field and Sample of Data Grid

The moment due to the Earth's magnetic field can be modeled as

$$
\boldsymbol{\tau}=\boldsymbol{\mu} \times \mathbf{B}
$$

where

$\tau=$ resulting torque applied to Spartnik (dyne $\cdot \mathrm{cm}$ )

$\mu=$ magnetic dipole moment of Spartnik (EMU)

$\mathbf{B}=$ local magnetic field vector of Earth's magnetic field (Gauss)

The required magnetic dipole of the magnets on Spartnik have been preliminary estimated to be $5.2 \times 10^{3}$ EMU for two magnets, directed in the positive $b_{3}$ direction. The strength of these magnets will not vary significantly within the life span of Spartnik due to their low demagnetization properties ${ }^{6}$.

Now that both parameters for equation (15) are defined the torque that Spartnik will experience through its orbit due to the Earth's magnetic field and the permanent magnets can be computed by the process described below:

(1) Calculate the position of Spartnik on its orbit using Kepler's Equation.

(2) Compute the latitude and longitude of Spartnik's center of mass.

(3) Use the latitude, longitude, and bilinear interpolation to calculate the local magnetic field vector (B)
(4) Calculate $\mu$. The direction of the vector, in the rotating frame, is determined by Spartnik's current orientation.

(5) Calculate $\tau$ and integrate EOMs to get new orientation of Spartnik.

$\left(\right.$ Note $\left.\mathbf{M}=\tau=\mathbf{M}_{1} \mathbf{b}_{\mathbf{1}}+\mathbf{M}_{2} \mathbf{b}_{2}+\mathbf{M}_{3} \mathbf{b}_{3}\right)$

\section{Numerical Simulation Methodology}

MATLAB was the chosen tool to numerically integrate the EOMs described above. The reason for this choice was ease of development and familiarity of the program by the authors. For the purpose of debugging the code the function "ode23" was used to numerically integrate the EOMs. Ode23 uses second and third order Runga-Kutta formulas to numerically integrate a system of ordinary differential equations. While debugging the code the tolerance of the integration was set at $1 \times 10^{-6}$. When the simulation was fully debugged fourth and fifth order Runga-Kutta formulas were used with the "ode 45 " function call and the tolerance lowered to $1 \times 10^{-12}$.

\section{Results}

One of the goals of the simulation is to determine if the passive control system, as initially designed, will perform as planned. If the passive control system does perform as planned then simulations can be performed to determine how well it works under a variety of conditions. If it does not perform as planned then simulations can be run under a variety of conditions with the goal of making recommendations on how to improve the passive control system design. If Spartnik performs as expected it should tumble about the $\mathbf{y}$ axis at a rate of 720 degrees per orbit or two complete tumbles per orbit. The rotating frame will complete one revolution per orbit. Therefore, Spartnik should complete one tumble per orbit with respect to the rotating frame. All simulations were run over a half of an orbit, beginning over the North Pole and ending near the South Pole. Thus, Spartnik should tumble (pitch) through 90 degrees in a quarter orbit. The spin rate (yaw) should be constant and reflected in the output as a line of constant, increasing slope equal to the spin rate. As described earlier the pitch offset $(\theta)$ of the body frame with respect to the rotating frame is limited to the range $-90^{\circ}$ to $+90^{\circ}$. However, over a half orbit Spartnik should pitch through 180 degrees. Therefore, in order to describe orientations of the satellite when it has "tumbled" through more than 90 degrees a 180 degree change in roll $(\phi)$ is kinetically required. When this 180 degree change in $\phi$ occurs, at the equator, $\theta$ will reach 90 degrees and then "rebound," rising back to zero. 
With the equations of motion and the external moments defined, the MATLAB program was run through a series of verification checks to make sure the program was working correctly and free of bugs. As stated earlier, initially the simulation was run using ode 23 with a tolerance of $1 \times 10^{-6}$. After the bugs had been eliminated from the code the simulation was run using ode 45 and the tolerance lowered in steps down to a final value of $1 \times 10^{-12}$. Although it is possible to lower the tolerance even more, the authors felt that this might induce round-off errors that could significantly change the results due to the low step size. All results discussed below were computed using ode 45 with a tolerance setting of $1 \times 10^{-12}$

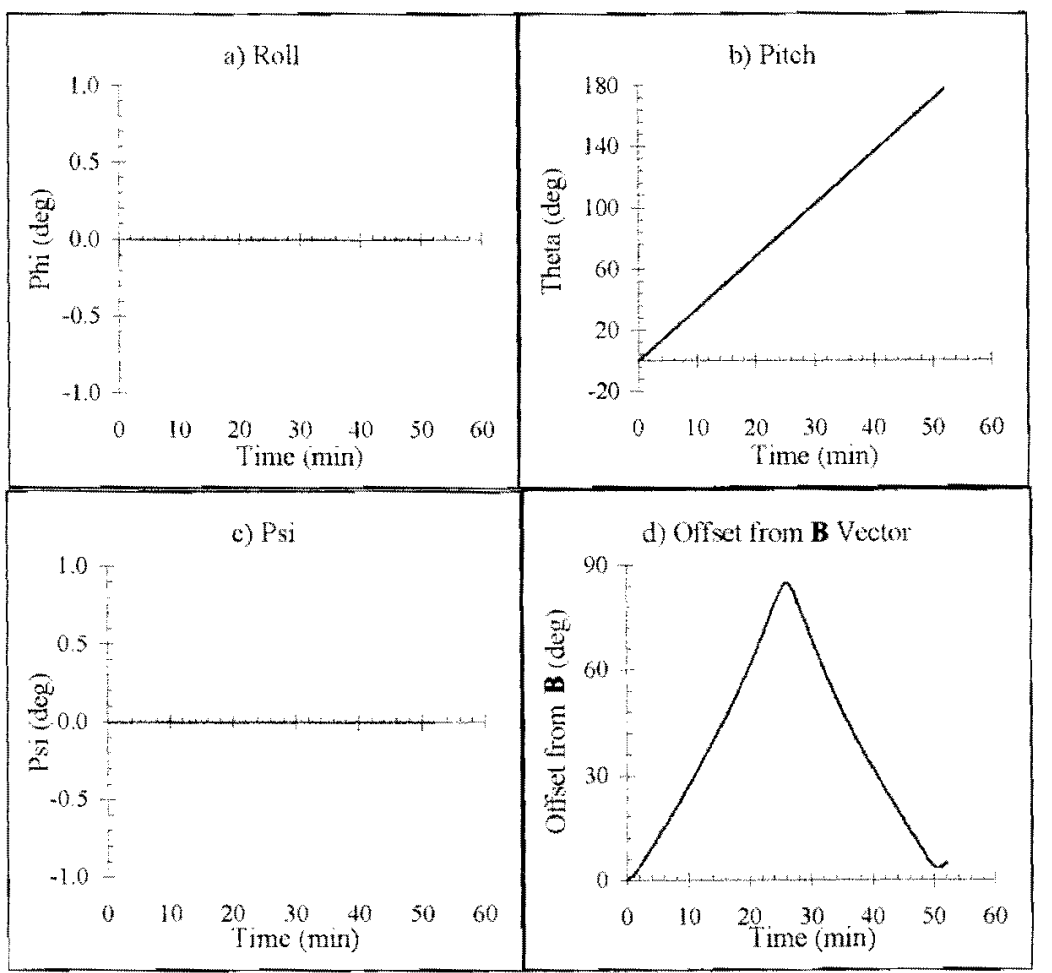

Figure 8(a-d): First Program Verification Simulation

The results confirm that Spartnik remains inertially pointed throughout the simulation. The rotating frame, however, does not remain inertially pointed and rotates as Spartnik moves along its orbit with a speed equal to the mean motion along the negative $\mathbf{y}$ direction. Therefore, Spartnik will appear to rotate along the positive $y$ direction with respect to the rotating frame. This can be seen in Figure $8 \mathrm{~b}$.

The second verification performed involved setting the magnet strength to its initial values as determined by the original control system design. This involved two magnets whose combined magnetic dipole is equal to $5.2 \times 10^{3}$ EMU. All other initial conditions, including spin rate, were set to zero (i.e. $\omega_{x}=0.0, \omega_{y}=$

\section{Program Verification}

Two simulations were run in order to verify the program was working correctly. The first involved setting the initial spin rate and magnet strengths to zero. This has the effect of simulating Spartnik as inertially pointed in space. With no external torques Spartnik should remain inertially pointed. All initial conditions for this simulation were set to zero (i.e. $\omega_{\mathrm{x}}=0.0, \omega_{\mathrm{y}}=0.0$, $\omega_{2}=0.0, \dot{\phi}=0.0, \dot{\theta}=0.0, \dot{\psi}=0.0$ ). The results of this simulation are depicted in Figure 8a-d. 


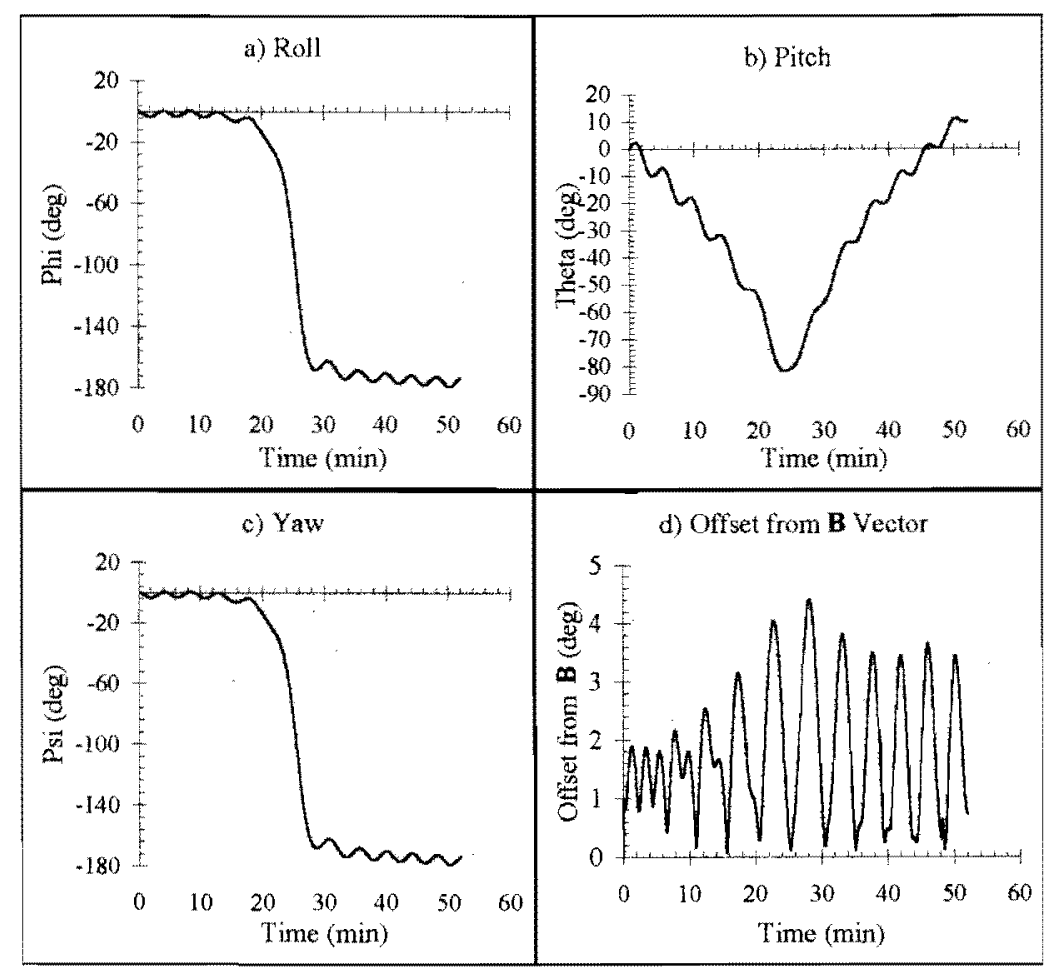

Figure 9 (a-d): Second Program Verification Simulation

\section{Gyroscopic Effect Simulations}

With the simulation working as expected it was determined to investigate the gyroscopic effect and determine if the control system, as designed, would perform as desired. The next simulation performed involved setting the spin rate about the $\mathbf{b}_{3}$ axis to 0.5

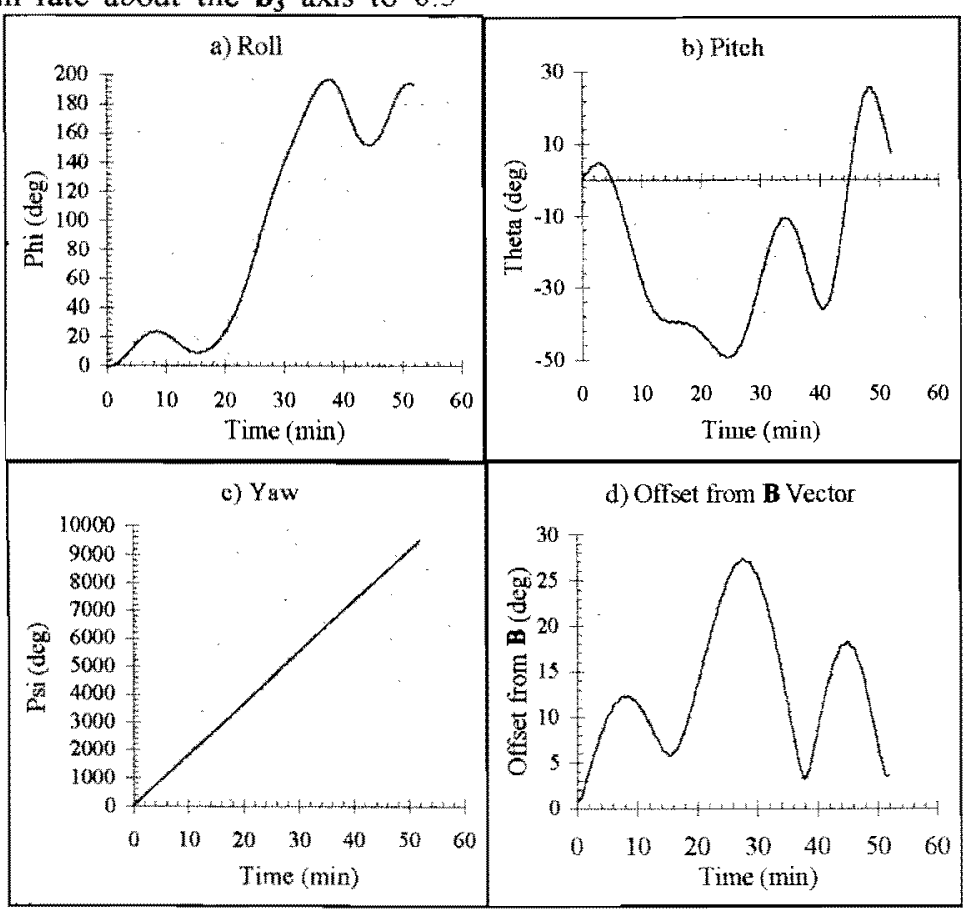

Figure 10 (a-d): Current Control System Simulation Results revolutions per minute $(\dot{\theta}=0.05236 \mathrm{radians} / \mathrm{sec}$, all others zero) and magnet strength of $5.2 \times 10^{3}$ EMU. These conditions represent the steady state conditions that are desired in orbit with the current control system. The results of the simulation are summarized in Figure $10(a-d)$. 
As can be seen from Figure 10b Spartnik does nor tumble the desired 90 degrees in a quarter orbit. The offset from the Earth's magnetic field, depicted in Figure $10 \mathrm{~d}$, grows unacceptably high. By the end of a quarter orbit the offset is over 25 degrees. This indicates that the gyroscopic effect is preventing Spartnik from locking onto the Earth's magnetic field and therefore not tumbling the desired amount.

The next series of simulations continued the investigation of the gyroscopic effect. The results depicted in Figure 10 (a-d) indicate that the magnets are either too weak to lock Spartnik onto the Earth's magnetic field, the satellite is spinning too fast, or, more probably, a combination of the two. Although simulations were run over a half orbit, the number of degrees tumbled in a quarter orbit will be used as the test for how well the system behaves. This is due to the complex motion in pitch and roll. Recall, the pitch should tumble through near 90 degrees over a quarter orbit and then "rebound". Over the next few simulations the spin rate was lowered incrementally until the magnets were able to tumble the satellite over 90 degrees in a quarter orbit. The results of these simulations are summarized below:

\section{Table 2: Investigation of Spin Rate on Control System} Performance

\begin{tabular}{|c|c|c|}
\hline $\begin{array}{c}\text { Spin Rate } \\
\text { (rev/min) }\end{array}$ & $\begin{array}{c}\text { Degrees } \\
\text { Tumbled in } \\
1 / 4 \text { Orbit } \\
\text { (degrees) }\end{array}$ & $\begin{array}{c}\text { Maximum } \\
\text { Offet from B } \\
\text { Vector } \\
\text { (degrees) }\end{array}$ \\
\hline 0.5 & 50 & 28 \\
\hline 0.4 & 62 & 38 \\
\hline 0.3 & 80 & 27 \\
\hline 0.2 & 84 & 12 \\
\hline 0.1 & 90 & 8 \\
\hline
\end{tabular}

For spin rates below 0.2 revolutions per minute the satellite tumbles the desired amount. Remember, because Spartnik may not be perfectly "locked" onto the Earth's magnetic field the satellite may not tumble through the complete 90 degrees in a quarter orbit. The total number of degrees may be less than 90 if the offset from the Earth's magnetic field is large. Nevertheless an offset from the Earth's magnetic field of under 10 degrees is acceptable for Spartnik. So, if the satellite tumbles through approximately 80 degrees in a quarter orbit it is assumed the passive control system is working correctly. At $0.2 \mathrm{revs} / \mathrm{min}$ the maximum offset from the Earth's magnetic field was 19 degrees.

\section{Magnet Strength Simulations}

The next series of simulations involved investigating the strength of the magnets and their effect on control system performance. Eight simulations were run each incrementing the number of magnets, and therefore, the total magnetic dipole magnitude. The spin rate for each of these simulations was set at 0.5 revolutions per minute. The results of these simulations are summarized below

Table 3: Investigation of Magnet Dipole Strength on System Performance

\begin{tabular}{|c|c|c|c|}
\hline $\begin{array}{c}\text { Number of } \\
\text { Magnets }\end{array}$ & $\begin{array}{c}\text { Total Magnetic } \\
\text { Dipole (EMU) }\end{array}$ & $\begin{array}{c}\text { Degrees } \\
\text { Tumbled in } \\
1 / 4 \text { Orbit } \\
\text { (degrees) }\end{array}$ & $\begin{array}{c}\text { Maximum } \\
\text { Offset from B } \\
\text { Vector } \\
\text { (degrees) }\end{array}$ \\
\hline 2 & $5.2 \times 103$ & 74 & 26 \\
\hline 4 & $1.04 \times 10^{4}$ & 85 & 12 \\
\hline 6 & $1.56 \times 10^{4}$ & 86 & 9.8 \\
\hline 8 & $2.08 \times 10^{4}$ & 87 & 7.8 \\
\hline
\end{tabular}

From Table 3 it can be seen that a total magnetic dipole of $1.04 \times 10^{4} \mathrm{EMU}$ is required in order to overcome the gyroscopic effect induced by the 0.5 $\mathrm{min} / \mathrm{rev}$ spin and cause Spartnik to tumble as desired. The maximum offset from the Earth's magnetic field vector was 12 degrees at this magnetic dipole strength. One interesting effect of increased magnetic dipole was an oscillation of the offset angle from the Earth's magnetic field. Because the magnetic dipole is so strong any offset from the Earth's magnetic field will cause a relatively large torque which, in turn, will quickly rotate the satellite in the direction of the field vector. The stronger the magnets the higher frequency this oscillation became.

\section{Moments of Inertia Simulations}

The final series of simulations run involved changing the moments of inertia in order to determine what effect this would have on the performance of the control system. The current design of Spartnik calls for ballast, in the form of metal plates, to be added to the outer shell of Spartnik in order to make $I_{2 z}$ the largest moment of inertia. However, the amount of ballast has yet to be determined. By varying the moments of inertia the simulation can provide some insight into the amount of ballast required for optimal performance of the control system under a variety of conditions. Moments of inertia are also difficult to determine precisely so information on how any possible errors in their calculation will affect the control system is also important. 
During this series of simulations the spin rate was set at 0.5 revolutions per minute and the magnet dipole strength at $5.2 \times 10^{3}$ EMUs. The moment of inertia about the $\mathrm{z}$ axis $\left(\mathrm{I}_{7 z}\right)$ was incrementally decreased from $0.331646 \mathrm{~kg} / \mathrm{m}^{4}$, corresponding to 1.4 times $I_{y y}$, to $0.23689 \mathrm{~kg} / \mathrm{m}^{4}$ corresponding to 1.1 times $\mathrm{I}_{\mathrm{yy}} . \mathrm{I}_{\mathrm{xx}}$ and $\mathrm{I}_{\mathrm{yy}}$ were held constant. The results of the simulation are summarized below:

Table 4: Investigation of Varying Moments of Inertia

\begin{tabular}{|c|c|c|c|}
\hline $\begin{array}{c}\mathrm{I}_{z z} \\
\left.\text { (fraction of } \mathrm{I}_{\mathrm{yy}}\right)\end{array}$ & $\begin{array}{c}\text { Degrees } \\
\left(\mathrm{kg} \mathrm{m}^{2}\right)\end{array}$ & $\begin{array}{c}\text { Maximum } \\
\text { Tumbled in } \\
1 / 4 \text { Orbit } \\
\text { (degrees) }\end{array}$ & $\begin{array}{c}\text { Offset from B } \\
\text { Vector } \\
\text { (degrees) }\end{array}$ \\
\hline 1.4 & 0.45 & 52 & 50 \\
\hline 1.3 & 0.42 & 55 & 45 \\
\hline 1.2 & 0.38 & 62 & 38 \\
\hline 1.1 & 0.35 & 68 & 33 \\
\hline 1.0 & 0.32 & 75 & 27 \\
\hline
\end{tabular}

As $I_{2 x}$ decreases it is expected that the gyroscopic stiffness of the system should also decrease and the performance of the magnets and the system as a whole should increase. From the results of this simulation this appears to be happening. However, Spartnik never achieves the desired tumble of 90 degrees over a quarter orbit. Varying the moments of inertia does not have as strong an effect on the performance of the control system as does varying the magnet strength or the spin rate. However, these simulations provide

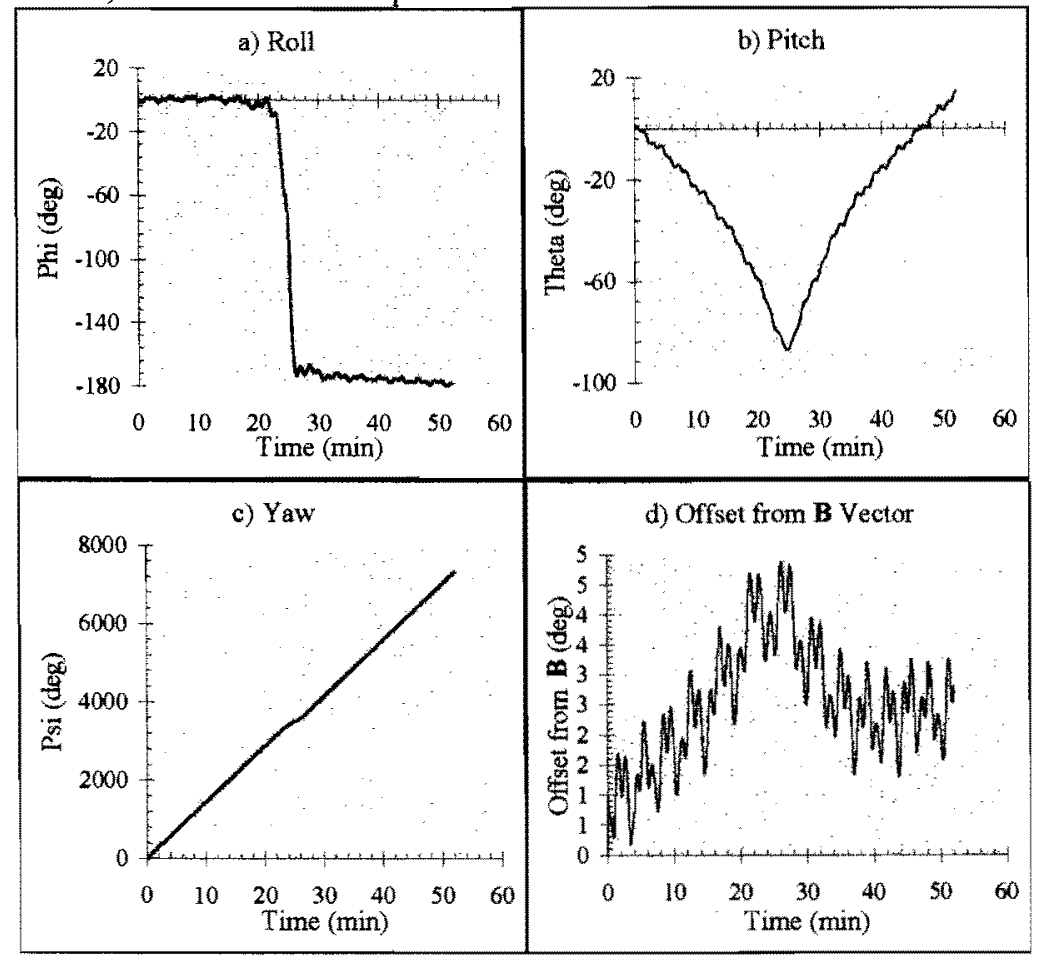

Figure 11(a-d): Modified Control System Simulation Results useful information that will be needed when selecting the final design of the passive control system.

\section{Modified Control System Design}

The results of the simulations raise concerns about the current control system design for Spartnik. However, they also provide some insight into what changes are necessary to improve the control system and make it more robust. The three parameters, spin rate, moments of inertia, and magnet dipole strength, all interact and make finding a simple solution for the control system difficult. The most difficult of these parameters to control is the spin rate. The factors that determine spin rate, namely: area of solar pressure paddles, difference in reflectance of both sides, amount and size of hysteresis rods, are known. However, calculating an accurate and reliable spin rate is very difficult. Therefore, it seems reasonable to determine a control system by changing the magnet strength and moments of inertia to suit a wide range of possible spin rates. Any results gained by these simulations that would determine magnet strength and moments of inertia must be checked against other subsystems. If the magnets are made too strong they can interfere with computer memory and possibly communications. The addition of mass along the outer shell of Spartnik must be checked for size constraints. 
Taking the above factors into account a new control system was designed for Spartnik. The new design was driven by a need to overcome the gyroscopic effect while not knowing precisely what the final spin rate of Spartnik will be. Therefore, every effort was made to change the design to lower the gyroscopic stiffness. Specifically, the number of magnets was increased from 2 to 8 magnets, increasing the total magnetic dipole from $5.2 \times 10^{3}$ EMU to $2.8 \times 10^{4}$ EMU. The moment of inertia about the $z$-axis was lowered from an estimate of 1.5 times $\mathrm{I}_{\mathrm{yy}}$ to 1.1 times $\mathrm{I}_{\mathrm{y} y}$. Finally, the difference between the reflective and absorptive sides of the solar pressure paddles will be changed in an attempt to lower the spin rate. A conservative estimate of the new spin rate is $0.4 \mathrm{rev} / \mathrm{min}$, down from $0.5 \mathrm{rev} / \mathrm{min}$. The results of this new control system design over a half orbit for a $1000 \mathrm{~km}$ altitude are depicted in Figure 11 (a-d). As can be seen from Figure $1 \mathrm{lb}$ Spartnik tumbles through 90 degrees and then "rebounds" back towards zero. The offset from the Earth's magnetic field vector, depicted in Figure 11d, remains close to zero over the entire simulation indicating that Spartnik is "locked" onto the Earth's magnetic field. Finally, $\psi$ remains close to zero when Spartnik is in the northern hemisphere. When $\theta$ reaches $90^{\circ}, \psi$ drops to $-180^{\circ}$ and remains there for the remainder of the simulation. Therefore, this control system design meets all the design requirements.

\section{Conclusion}

All the simulations described above were run at an altitude of $1000 \mathrm{~km}$ and in a polar orbit. Since the actual orbit of Spartnik may be lower than $1000 \mathrm{~km}$ the performance of the modified control system at lower altitudes is also needed. However, since the magnetic field drops off with altitude, one can assume that if the control system performs adequately at $1000 \mathrm{~km}$, it will perform well at lower altitude. Additional investigations are needed for different inclinations.

Additionally, it must be remembered that any results gained by this simulation are just that, a simulation of reality. When Spartnik is in orbit there will be many more torques present, including solar pressure and gravity gradient torques. In addition, there will be dissipative forces that were not included in the simulation, including the hysteresis rods and the nutation damper; these will aid in the stabilization of Spartnik. These additional effects were unable to be included in this model due to time limitations. As mentioned earlier, the magnitudes of the torques described above will be orders of magnitude less than that of the magnets interaction with the Earth's magnetic field. However, their ultimate effect on the control system will be seen when Spartnik is launched into orbit.

\section{Acknowledgments}

The authors would like to thank the following people and organizations: the Mechanical and Aerospace Engineering Department for giving us the opportunity to participate in this project, Emily Lewis for her help throughout this project and in writing this paper, Professor Parvin of the SJSU Physics Department for helping us develop the magnetic modeling of Spartnik and its interaction with the Earth's magnetic field. Sean Hwang for his help in deriving the frames and equations of motion. And finally, a special word of appreciation to Professor Pernicka for providing us with guidance, helping us overcoming the many difficulties that arose, and for reading every revision of this paper

\section{References}

1. Bonsall, Charles A., (editor) We hersat Users Handbook, Weber State University, 1991

2. White, Jim, MicroSat Motion, Stabilization and Telemetry, presented at the Small Satellite Conference, Logan, Utah, 1990.

3. Larson, W.J and Wertz, J.R., (editors) Space Mission Analysis and Design, Microcosm, Inc, Torrance, CA and Kluwer Publishers, The Netherlands, 1992.

4. Spartnik: San José State University's MicroSatellite Project, Department of Mechanical and Aerospace Engineering, SJSU, Dec 1996.

5. King, J.H (king@nssdca.gsfc nasa.gov), DGRF IGRF Geomagnetic Field Model 1945 2000 and Related Parameters, http://nssdc.gsfc.nasa.gov/space/model/models/igrf. html, December 1995.

6. The Dexter Corporation, Permanent Magnet Catalog, Dexter Magnetic Materials Division, 1992.

7. Press, William H, et., al., eds, Numerical Recipes in C: The Art of Scientific Computing, Cambridge University Press, Cambridge, NY, 1988. 


\footnotetext{
1 Webersat's document

${ }^{2}$ Jim White Microsatellite

${ }^{3}$ Wertz

${ }^{4}$ SJSU FDR

${ }^{5}$ Web Site

${ }^{6}$ Dexter Catalog
} 